\title{
Capacity Estimation for On-Ramp Merging Section of Urban Expressway Based on Time Headway Loss
}

\author{
Xing-jian Xue, ${ }^{1,2,3}$ Feng Shi, ${ }^{1}$ and Qun Chen ${ }^{1}$ \\ ${ }^{1}$ School of Transportation Central South University, Changsha 410075, China \\ ${ }^{2}$ Hunan Province University Key Laboratory of Bridge Engineering (Changsha University of Science \& Technology), \\ Changsha 410000, China \\ ${ }^{3}$ School of Transportation and Logistics Central South University of Forestry and Technology, Changsha 410004, China \\ Correspondence should be addressed to Qun Chen; chenqun631@csu.edu.cn
}

Received 25 September 2013; Revised 31 December 2013; Accepted 1 January 2014; Published 20 February 2014

Academic Editor: Huimin Niu

Copyright (C) 2014 Xing-jian Xue et al. This is an open access article distributed under the Creative Commons Attribution License, which permits unrestricted use, distribution, and reproduction in any medium, provided the original work is properly cited.

\begin{abstract}
This paper proposes a model for estimating capacity of on-ramp merging section of urban expressway based on dynamics and gap acceptance theory, considering lane-changing processes and time headway loss. Survey data were collected from on-ramp merging sections of shanghai urban expressway system and showed that capacity drop of on-ramp merging section is caused by drivers' lanechanging which may lead to unsteady speed of vehicles and so prolonged time headway compared to the minimum time headway corresponding to the maximum capacity. Three parameters (optimal time headway, time headway loss, and interference quantity of lane-changing) are given and a methodology by accumulating time headway loss due to lane-changing is developed to estimate the capacity drop. Results' comparisons between real data and microsimulation of on-ramp merging sections and sensitivity analysis show that the proposed model can produce reliable and accurate results. This study also reveals that ramp flow and the difference between the optimal speed and the lane-changing speed of fleet have a great impact on capacity drop. This study is beneficial to evaluate congestion levels, to understand complex traffic phenomena, and so to find efficient solutions.
\end{abstract}

\section{Introduction}

Traffic congestion causes a huge disadvantage, such as waste of time and fuel as well as pollution. On urban expressways, congestion is usually induced by various kinds of bottleneck, among which the on-ramp merging section is an important position and has been widely studied. To enhance the capacity of an expressway with an on-ramp merging section, various kinds of methods have been proposed, in which an accurate traffic model that can efficiently estimate the capacity of on-ramp is most important while evaluating congestion levels, understanding complicated traffic phenomena, putting forward reasonable design scheme of infrastructure, and developing efficient operation strategies.

In the past decades, continuous effort has been devoted to the study of capacity of on-ramp merging section. Many researchers have tried to identify the causes of capacity drop at on-ramp merging section that seems to be linked to flow interference, which are created by vehicle lane-changing maneuvers [1-6].

Several models have been proposed to account for capacity. The most commonly studied model is probabilistic model based on gap acceptance theory. The gap acceptance theory was put forward by Drew [7]. Westphal concluded that the on-ramp capacity decided by outmost lane flow [8]. Lertworawanich and Elefteriadou developed a new gap acceptance model through definition expressway capacity as function of ruin probability [9]. Kim and Son promote a new model of on-ramp capacity through making time headway obey different flow range [10], but most of these models are hard to implement and to calibrate in practice $[2,11]$. However, the development of new tools now allows having access to more refined information and more rapid calculations [10]. In contrast, the method put forward by HCM based on regression analysis not only is simple but also has very strong practicability [10]; its disadvantage is high 
cost of data investigation and small scope of application. In addition, there are traffic wave capacity model, cell automaton capacity model, and so forth [10]. Recent studies have shown that the capacity of expressway is not immutable and frozen; many researchers suggest that capacity can only truly be defined as a function of breakdown probability $[12,13]$.

The overall objective of this study is to develop a new onramp merging section capacity estimation model of urban expressway, which considers lane-changing processes, vehicle speed of on-ramp, vehicle flow, and time headway distribution. At first, a new modeling method about capacity is discussed and some new parameters were proposed. Then, this paper develops a series of hypotheses about capacity model, and the study site and traffic data in detail have been described. Section 3 builds the capacity model about on-ramp merging section of urban expressway, and the parameters were calibrated. In the final section, the data obtained from the capacity model are compared with field-observed capacities and microsimulation result, and sensitivity analyses show that our model is robust; the implications of the research results are discussed in different fields.

\section{Idea of Mathematical Modeling and Its Theoretical Explanation}

The traffic flow is inversely proportional to the time headway of vehicles. Without lane-changing, time headway is related to some factors such as speed, traffic flow interference, and the reaction time of drivers. The time headway reaches a minimum value when capacity reaches a maximum value. The minimum time headway is just the optimal headway, symbolized as $V_{o}$, and the corresponding speed is the optimal speed, symbolized as $V_{o}$.

The relationship between the flow and the velocity can be derived from the Greenshields model, and its curve of relationship is shown in Figure 1(a). The figure shows that the optimal speed corresponds to the minimum time headway. However, because in most cases the real situation cannot correspond to the results obtained from the model well, the relation between speed and time headway should be derived from measured data. In this paper, the scatter diagram (shown in Figure 1(b)) about the relation of speed and headway was obtained from using one week 5 min loopdetector data at Shanghai expressway system. The position of data investigation is the inner lane of Yan'an road of the Shanghai expressway system because here the traffic flow has the most stable state and the least merging interference. The survey data show that the capacity can reach a maximum value $(2050 \mathrm{pcu} / \mathrm{h})$ that corresponds to the minimum time headway $(1.8 \mathrm{~s})$ when the speed keeps about $45 \mathrm{~km} / \mathrm{h}$, as shown by Figure 1(b); the result is consistent with the actual situation. Therefore, this paper defines $2000 \mathrm{pcu} / \mathrm{h}$ as the capacity per one lane, the $1.8 \mathrm{~s}$ as the optimal headway, and $45 \mathrm{~km} / \mathrm{h}$ as the optimal speed.

On-ramp merging sections usually become the bottleneck of urban expressway because headway cannot keep an optimal value; namely, the time headway is prolonged due to unsteady traffic flow caused by lane-changing of vehicles

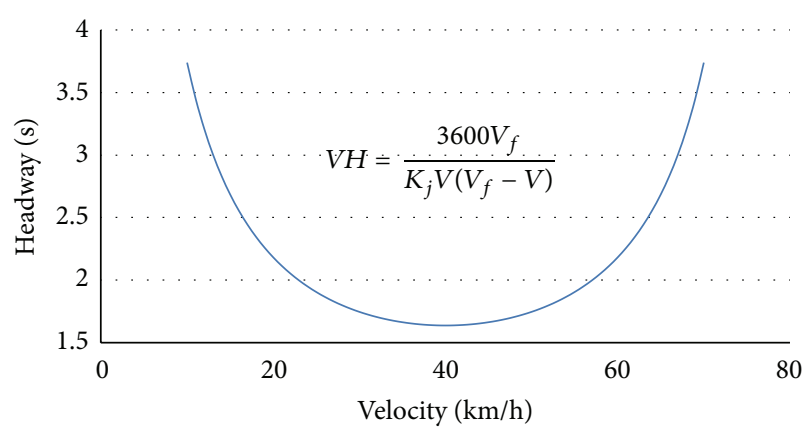

(a) The model of Greenshields

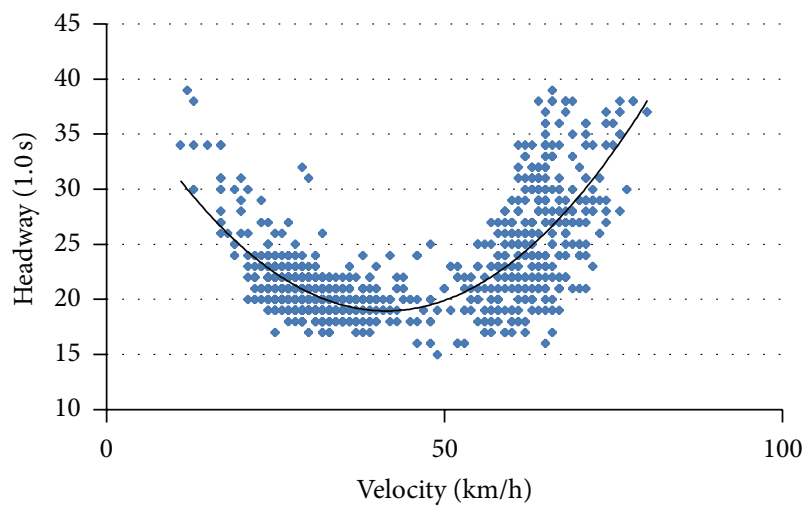

(b) The fitting of the measured data

FIGURE 1: The relation between time headway and vehicle speed.

and so frequently variable speed. The prolonged part of time headway from lane-changing can be used for more vehicles passing and is called as the single loss of time headway, symbolized as $T_{l}$. The operational state of vehicle at onramp merging section is shown in Figure 2(a). When the vehicle running on the most medial lane passes the on-ramp merging section with a speed of $V_{o}$, whose corresponding time headway is $V H_{o}$, the time headway of vehicles running on the outermost lane would prolong due to interferes from flows merging. The fleet of different quantity scale would be merging to mainline according to gap size between vehicles running on the most lateral lane. For example, fleet 1 consists of one car; fleet 2 consists of two cars, as shown in the Figure 2(b). The speed of vehicles running on the mainline behind fleet 1 is forced to slow down to $V_{1}$ because of the interference of fleet 1's lane-changing to the mainline with this speed. So, a larger headway $V H_{1}$ appears between vehicle 11 of fleet 1 as head car and vehicle 2 . It is the loss of travel time $\left(t_{l}\right)$ for lane-changing of fleet 1 which is used for speed 1 arising to speed 2 without considering other factors such as drivers' reaction time and internal interference, the same reason for analysis of fleet 2 . The number of merging fleet in one unit of time is called as the interference quantity of lanechanging, denoted by $q_{r g}$. The interference quantity of lanechanging is not equal to the vehicle number of lane-changing; it is equal to the number of major road gap that is greater than critical gap of lane-changing. For example, there are 10 ramp vehicles; if each gap of major road can only merge one 


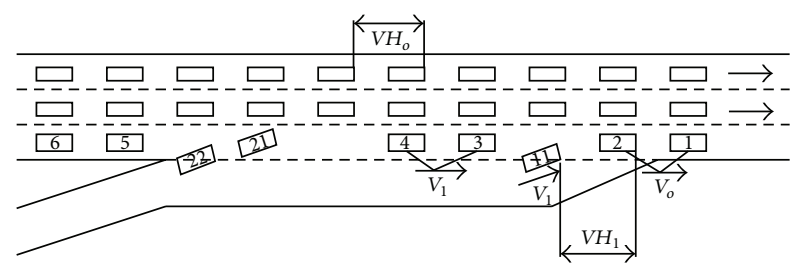

(a)

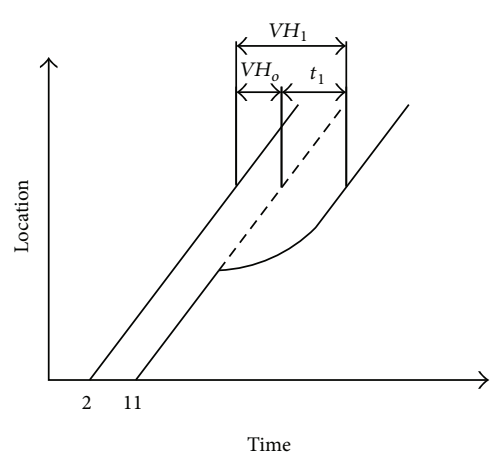

(b)

FIgURE 2: Traffic flows at on-ramp merging section.

vehicle, then the interference quantity of lane-changing is 10 ; if each gap of major road can merge two vehicles, then the interference quantity of lane-changing is 5 . Because there will only be one interference in a gap of major road traffic flow, do not change with the scale of the merge fleet. The product of $q_{r g}$ and $t_{l}$ is called as the headway loss per unit time. The difference between unit time and the headway loss is called as the available travel time. The capacity of lateral lane can be obtained if the flows of ramp and upstream mainline of lateral lane have been known. Finally, the capacity of on-ramp merging section can be obtained from accumulation of all of the lanes of the expressway.

\section{The Capacity Model for On-Ramp Merging Section Based on Time Headway Loss}

3.1. The Description of Parameters and Variables. $a$ is the acceleration of lane-changing fleet; $C$ is the capacity of onramp merging section; $C_{I}$ is the capacity of inside lane; $C_{o}$ is the capacity of lateral lane of mainline; $i$ is the vehicle number of lane-changing fleet, $i \geq 1 ; l$ is the order of Erlang distribution; $m$ is the lane number of mainline; $n$ is the maximum number of vehicles in lane-changing fleet; $p\left(t_{v h}>\right.$ $t_{c}$ ) is the probability of $t_{v h}$ being greater than $t_{c} ; p\left[t_{c}+i t_{f}>t>\right.$ $\left.t_{c}+(i-1) t_{f}\right]$ is the probability of headway $t$ between $t_{c}+i t_{f}$ and $t_{c}+(i-1) t_{f} ; q_{m}$ is the rate of flow in lateral lane of mainline upstream; $q_{r}$ is the vehicle number of lane-changing; $q_{r g}$ is the interference quantity of lane-changing; $T_{a}$ is the time for vehicles' acceleration from lane-changing speed to the optimal speed; $T_{b}$ is the basic loss headway, namely, the loss headway due to driver reaction time and internal interference of fleet; $T_{l}$ is the loss headway per lane-changing of the fleet; $t_{v h}$ is time-headway; $t_{c}$ is critical gap of lane-changing; $t_{f}$ is the headway of car-following; $t_{0}$ is the minimum headway;
$V_{o}$ is optimal speed; $V_{r}$ is the speed of vehicles running on the on-ramp; $V_{c}$ is the difference between the optimal speed and the lane-changing speed of fleet (speed difference of lanechanging for short); $V H_{o}$ is optimal headway; $\lambda$ is arriving rate of vehicles.

3.2. Basic Hypothesis. The model is based on some assumptions, including the following.

(1) The same type of vehicles has the same critical gap.

(2) The speed is consistent when these vehicles change lanes.

(3) The vehicle running on the ramp could change to mainline only if the gap of mainline is greater than the critical gap $\left(t_{c}\right)$.

(4) The effects of each lane-changing caused by interference will not spread to the upstream.

(5) The on-ramp traffic demand is saturated.

3.3. Model Construction. The capacity model of on-ramp merging section is shown in formula (1). It consists of two parts. The first part is the capacity of lateral lane of mainline, and its interference by lane-changing vehicle is shown in formula (2). The second part is the capacity of inside lane which is not disturbed by lane-changing vehicles, as shown in formula (3). Consider

$$
\begin{gathered}
C=C_{o}+C_{I}, \\
C_{o}=\frac{3600-T_{l} q_{r g}}{V H_{o}}, \\
C_{I}=\frac{3600}{V H_{o}}(m-1) .
\end{gathered}
$$

Parameter $T_{l}$ consists of the acceleration time of driving on-ramp $\left(T_{a}\right)$ and the loss headway due to internal interference of fleet $\left(T_{b}\right)$, as shown in formula (4). The size of acceleration time $\left(T_{a}\right)$ is related to the speed difference between $V_{o}$ and $V_{r}$, as well as the acceleration of fleet $(a)$ as shown in Figure 2(b). Their relationship is shown in formula (5). Consider

$$
\begin{gathered}
T_{l}=T_{a}+T_{b}, \\
T_{a}=\frac{\left(V_{o}-V_{r}\right)}{a} .
\end{gathered}
$$

Parameter $q_{r g}$ refers to the interference number due to lane-changing, and it is related to flow of lateral lane of expressway. Its meaning is different from $q_{r}$. The gap size distribution in different flow conditions is also different. When the flow is small, the size of gap is so small that the merging fleet is composed of only one car in most cases; thus the gap between $q_{r g}$ and $q_{r}$ is small. On the contrary, the gap is larger and the merging fleet consists of more vehicles. The $q_{r g}$ is the cumulative quantity of lane-changing fleet of different scale, and its model is shown in formula (6). Its depends on 


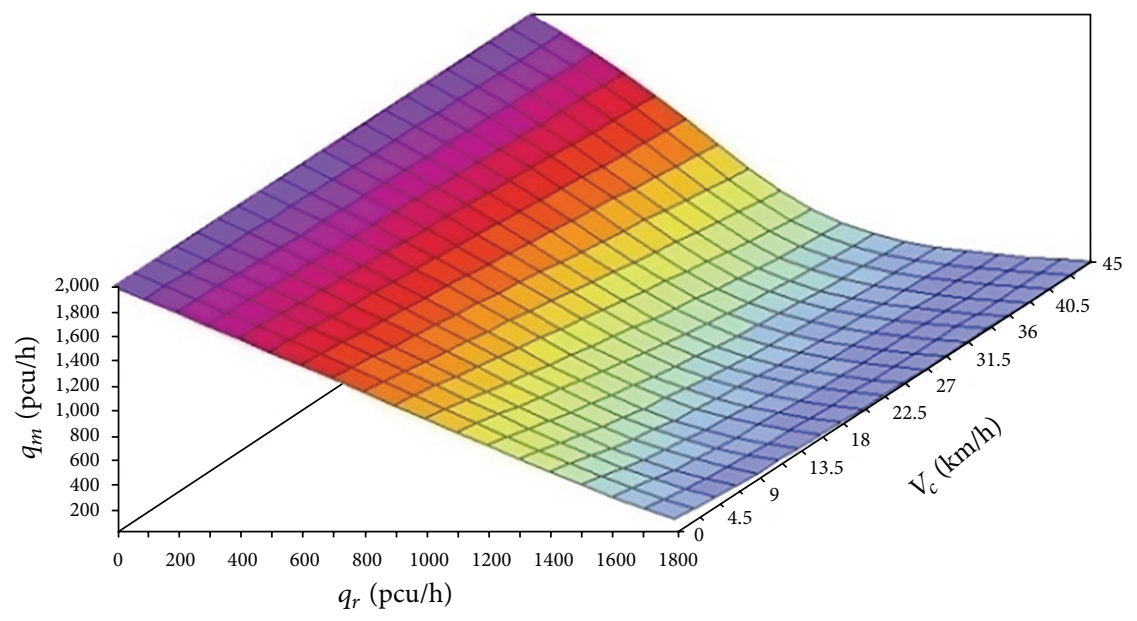

FIgURE 3: The relations between $q_{r}, V_{c}$, and $q_{m}$.

$t_{c}$ and $t_{f}$ about how large-scale fleet may merge to mainline in some headway. Consider

$$
q_{r g}=\sum_{i=1}^{n} \frac{p\left[t_{c}+i t_{f}>t_{v h}>t_{c}+(i-1) t_{f}\right] q_{r}}{p\left(t_{v h}>t_{c}\right) i} .
$$

Bringing formulas (5) (8) into formula (4), then the formula of on-ramp merging section capacity of urban expressway based on time headway loss is shown in the following formula:

$$
\begin{gathered}
C=\left(\left(3600-\left(\frac{\left|V_{o}-V_{r}\right|}{a}+T_{b}\right)\right.\right. \\
\left.\quad \times \sum_{i=1}^{n} \frac{p\left[t_{c}+i t_{f}>t_{v h}>t_{c}+(i-1) t_{f}\right]}{p\left(t_{v h}>t_{c}\right) i} q_{r}\right) \\
\left.\times\left(V H_{o}\right)^{-1}\right)+\frac{3600}{V H_{o}}(m-1) .
\end{gathered}
$$

The headway of lateral lane obeys different kinds of distributions with the flows changing. The Erlang distribution is suitable for describing headway distribution according to different flow conditions because it can describe different degree of flow conditions from unblocked to congestion by adjusting the order L, as shown in formula (8). The Erlang distribution about order 1 is the negative exponential distribution, and the headway will keep equal when the value of order $\mathrm{L}$ is very large. The research of the literature showed that the flow range of main lane in less than $700 \mathrm{pcu} / \mathrm{h}$, $700 \sim 1500 \mathrm{pcu} / \mathrm{h}$, and more than $1500 \mathrm{pcu} / \mathrm{h}$ was applied to the Erlang distribution of order 1, 2, 3 [14]. The usual value range of $t_{0}$ was $11.5 \mathrm{~s}$. Consider

$$
p\left(t_{v h} \geq t\right)=\sum_{i=0}^{l-1}(\lambda l t)^{i} \frac{e^{-\lambda l\left(t-t_{0}\right)}}{i !} .
$$

\subsection{Parameter Calibration}

(1) $t_{c}$ and $t_{f}$ : the literature research showed that the average value of $t_{c}$ is $3.0 \mathrm{~s}$ and $t_{f}$ is $1.5 \mathrm{~s}$ according to the survey based on 200 observation samples in some on-ramp merging sections of Beijing [15].

(2) $a$ : the $2 \mathrm{~m} / \mathrm{s}^{2}$ is a suitable value according to the mechanical properties of cars.

(3) $T_{b}$ : the $0.5 \mathrm{~s}$ is a suitable value according to survey data and microsimulation analysis.

(4) $n$ : although the vehicles in fleet per lane-changing may be more when the flow in the mainline's lateral lane is very small, the suggested value of $n$ is $4 \sim 8$ for computational simplicity and accuracy.

\section{Model Calculation}

The relations between $q_{r}, V_{c}, q_{m}$, and $C_{o}$ have been obtained by the model programming and solving using LM (Levenberg-Marquardt) algorithm, as shown in Figures 3 and 4 and Tables 1 and 2. As shown in Figure 3, the relation between $q_{r}$ and $q_{m}$ is linear, and the relation will be converted into nonlinear with the value of $V_{c}$ increasing. As shown in Figure 4, the main body of flow has changed from the vehicles driving on mainline to those driving on on-ramp with the increase of on-ramp vehicles. The capacity also showed a rising trend after the first drop. It showed that ramp traffic flow had a negative impact on the capacity of on-ramp merging section of expressway, and this negative effect was proportional to $V_{c}$.

As shown in Table 1, the capacity reached the lowest when the value of $q_{m}$ takes a value between $300 \sim 430 \mathrm{pcu} / \mathrm{h}$ and $q_{r}$ takes a value between $1000 \sim 1500 \mathrm{pcu} / \mathrm{h}$ with the increase of $V_{c}$. Table 2 shows that the maximum loss of capacity can reach $1 / 3$ of the total. This shows that it is very important that vehicles of ramp have enough acceleration space.

This model shows that the critical parameter for the capacity of on-ramp merging section is the $q_{r}$ and $V_{c}$. It is an effective way reducing the value of these two factors to 


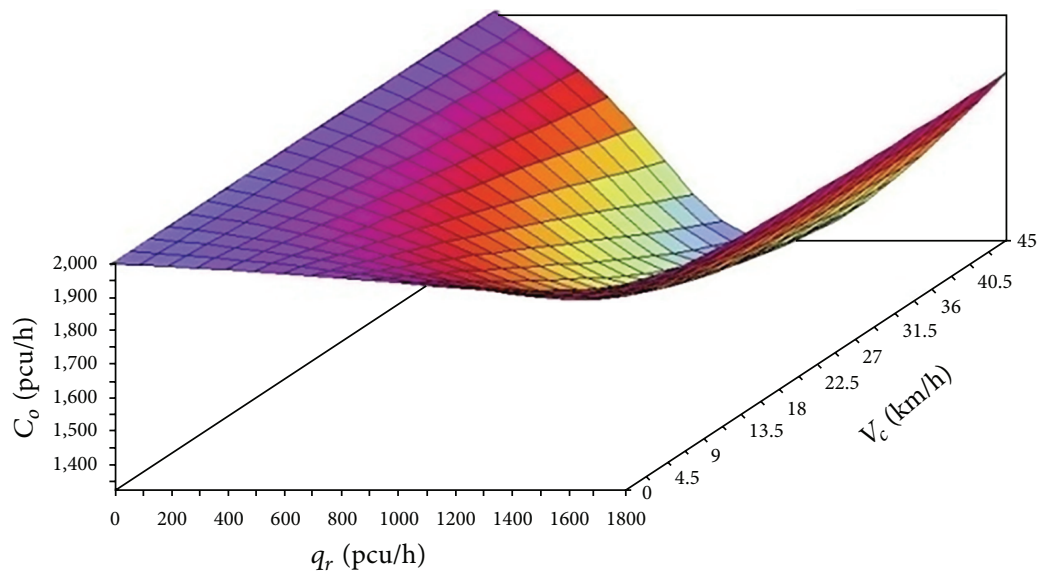

FIgURE 4: The relations between $q_{r}, V_{c}$, and $C_{o}$.

TABLE 1: The relations between $q_{r}, V_{c}$, and $q_{m}$.

\begin{tabular}{|c|c|c|c|c|c|c|c|c|c|c|c|}
\hline \multirow{2}{*}{$q_{m}(\mathrm{pcu} / \mathrm{h})$} & \multicolumn{11}{|c|}{$V_{c}(\mathrm{~km} / \mathrm{h})$} \\
\hline & 0 & 4.5 & 9 & 13.5 & 18 & 22.5 & 27 & 31.5 & 36 & 40.5 & 45 \\
\hline 200 & 1793 & 1783 & 1774 & 1764 & 1754 & 1745 & 1735 & 1725 & 1715 & 1704 & 1694 \\
\hline 400 & 1583 & 1562 & 1540 & 1518 & 1495 & 1472 & 1448 & 1423 & 1398 & 1373 & 1346 \\
\hline 600 & 1372 & 1336 & 1299 & 1261 & 1221 & 1180 & 1138 & 1094 & 1050 & 1004 & 957 \\
\hline 800 & 1159 & 1106 & 1051 & 995 & 936 & 876 & 815 & 755 & 695 & 637 & 583 \\
\hline 1000 & 945 & 874 & 802 & 728 & 655 & 586 & 520 & 461 & 409 & 363 & 325 \\
\hline 1200 & 731 & 645 & 561 & 482 & 412 & 352 & 303 & 263 & 230 & 204 & 183 \\
\hline 1400 & 521 & 429 & 349 & 284 & 234 & 197 & 168 & 146 & 129 & 115 & 104 \\
\hline 1600 & 320 & 241 & 185 & 147 & 120 & 101 & 87 & 76 & 68 & 61 & 55 \\
\hline 1800 & 141 & 98 & 73 & 58 & 48 & 41 & 35 & 31 & 28 & 25 & 23 \\
\hline$T_{l}(\mathrm{~s})$ & 0.00 & 0.63 & 1.25 & 1.88 & 2.50 & 3.13 & 3.75 & 4.38 & 5.00 & 5.63 & 6.25 \\
\hline
\end{tabular}

TABLE 2: The relations between $q_{r}, V_{c}$, and $C_{o}$.

\begin{tabular}{|c|c|c|c|c|c|c|c|c|c|c|c|}
\hline \multirow{2}{*}{$C_{o}(\mathrm{pcu} / \mathrm{h})$} & \multicolumn{11}{|c|}{$V_{c}(\mathrm{~km} / \mathrm{h})$} \\
\hline & 0 & 4.5 & 9 & 13.5 & 18 & 22.5 & 27 & 31.5 & 36 & 40.5 & 45 \\
\hline 200 & 1993 & 1983 & 1974 & 1964 & 1954 & 1945 & 1935 & 1925 & 1915 & 1904 & 1894 \\
\hline 400 & 1983 & 1962 & 1940 & 1918 & 1895 & 1872 & 1848 & 1823 & 1798 & 1773 & 1746 \\
\hline 600 & 1972 & 1936 & 1899 & 1861 & 1821 & 1780 & 1738 & 1694 & 1650 & 1604 & 1557 \\
\hline 800 & 1959 & 1906 & 1851 & 1795 & 1736 & 1676 & 1615 & 1555 & 1495 & 1437 & 1383 \\
\hline 1000 & 1945 & 1874 & 1802 & 1728 & 1655 & 1586 & 1520 & 1461 & 1409 & 1363 & 1325 \\
\hline 1200 & 1931 & 1845 & 1761 & 1682 & 1612 & 1552 & 1503 & 1463 & 1430 & 1404 & 1383 \\
\hline 1400 & 1921 & 1829 & 1749 & 1684 & 1634 & 1597 & 1568 & 1546 & 1529 & 1515 & 1504 \\
\hline 1600 & 1920 & 1841 & 1785 & 1747 & 1720 & 1701 & 1687 & 1676 & 1668 & 1661 & 1655 \\
\hline 1800 & 1941 & 1898 & 1873 & 1858 & 1848 & 1841 & 1835 & 1831 & 1828 & 1825 & 1823 \\
\hline$\overline{T_{l}(\mathrm{~s})}$ & 0.00 & 0.63 & 1.25 & 1.88 & 2.50 & 3.13 & 3.75 & 4.38 & 5.00 & 5.63 & 6.25 \\
\hline
\end{tabular}

improve the capacity of on-ramp merging section. There are two points of important significance. First, the value of $V_{c}$ can be reduced through setting the acceleration lane longer, but the measures must be taken to control on-ramp flow according to the situation of upstream flow of mainline's lateral lane and avoid the situation that on-ramp vehicles cannot change from ramp to mainline, which may lead to vehicles queuing on acceleration lane and so the space of acceleration cannot reach the expected. Second, the balance design of lane number of upstream and downstream of merging sections can decrease the value of $q_{r}$ because parts of on-ramp vehicles have no need of lane-changing. 
TABLE 3: The data comparison between model and actual measurement.

\begin{tabular}{|c|c|c|c|c|c|}
\hline \multirow{2}{*}{ Serial number } & \multirow{2}{*}{ Location } & \multirow{2}{*}{ Category } & \multirow{2}{*}{ Measured data $(\mathrm{pcu} / \mathrm{h})$} & \multicolumn{2}{|c|}{ Model calculation $(\mathrm{pcu} / \mathrm{h})$} \\
\hline & & & & Value & Error \\
\hline \multirow{2}{*}{1} & \multirow{2}{*}{ Wuyi road } & Capacity & 3631 & 3755 & $3.4 \%$ \\
\hline & & The flow of on-ramp & 742 & 742 & - \\
\hline \multirow{2}{*}{2} & \multirow{2}{*}{ Wuyi road } & Capacity & 3530 & 3732 & $5.7 \%$ \\
\hline & & The flow of on-ramp & 543 & 543 & - \\
\hline \multirow{2}{*}{3} & \multirow{2}{*}{ Wuning road } & Capacity & 3761 & 3613 & $-3.9 \%$ \\
\hline & & The flow of on-ramp & 1243 & 1243 & - \\
\hline \multirow{2}{*}{4} & \multirow{2}{*}{ Wuning road } & Capacity & 3701 & 3613 & $-2.4 \%$ \\
\hline & & The flow of on-ramp & 1259 & 1259 & - \\
\hline \multirow{2}{*}{5} & \multirow{2}{*}{ Jinshajiang road } & Capacity & 5212 & 5443 & $-4.1 \%$ \\
\hline & & The flow of on-ramp & 852 & 852 & - \\
\hline \multirow{2}{*}{6} & \multirow{2}{*}{ Jinshajiang road } & Capacity & 5287 & 5451 & $-3.0 \%$ \\
\hline & & The flow of on-ramp & 852 & 852 & - \\
\hline
\end{tabular}

\section{Comparison of Computational Results and Sensitivity Analysis}

5.1. Comparison of Computational Results. In order to test the accuracy of the results of model, this model calculation results are compared with the measured data of Shanghai expressway. These measured data are collected from inner ring of Shanghai urban expressway on-ramp merging section from 8:00 to 10:00, the cross section type of expressway is bidirectional and four lanes, and the length of all the acceleration lane is about 100 meters. The results of comparison are shown in Table 3. The error between model calculation and measured data is within 5\% according to the comparison in Table 3. In order to embody the relation between on-ramp flow and the flow of mainline lateral lane, this paper compares these data including VISSIM microsimulation and the model calculation results based on four kinds value of $V_{c}$, because there is not any road but only one lane in reality. The result of comparison show that there is a higher degree of fitting, as shown in Figure 5. This proved that the model has a high accuracy.

5.2. Sensitivity Analysis. In order to test whether the applicability of the model is better, sensitivity analysis carried out on the main parameters of the model are necessary. There are two parameters which have great influence on the model: $V H_{o}$ and $V_{c}$.

The meaning of sensitivity analysis of $V H_{o}$ is analysing the maximum capacity of lateral lane of expressway mainline. The change relation between $q_{r}$ and $C_{o}$ is shown in Figure 6 when the capacity value of lateral lane is $1800 \mathrm{pcu} / \mathrm{h}, 2200 \mathrm{pcu} / \mathrm{h}$, and $2400 \mathrm{pcu} / \mathrm{h}$. According to Figure 6, the relation between $q_{r}$ and $C_{o}$ basic keeps consistent with the increase of capacity. The maximum flow of on-ramp is increasing leading to the maximum drop of capacity with the increase of interference amplitude of on-ramp flow to the capacity of mainline. This is consistent with the actual situation because the aggrandizement of difference between on-ramp traffic flow and mainline traffic flow of expressway. The influence is increasing when vehicle lane is changing.

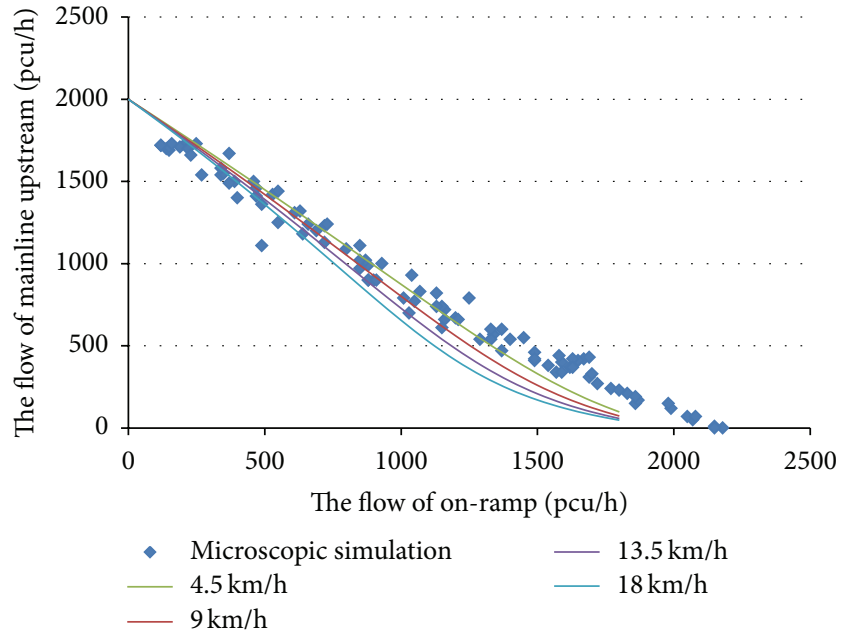

FIGURE 5: The data comparison between model and microscopic simulation of single lane.

The meaning of sensitivity analysis of $V_{c}$ expands the range of $V_{c}$, and its meaning to improving the value of the $V_{o}$. The value range of $V_{o}$ in this paper is from $45 \mathrm{~km} / \mathrm{h}$ to $80 \mathrm{~km} / \mathrm{h}$, and the corresponding value range of $V_{c}$ is from $45 \mathrm{~km} / \mathrm{h}$ to $80 \mathrm{~km} / \mathrm{h}$ (the value range from $0 \mathrm{~km} / \mathrm{h}$ to $45 \mathrm{~km} / \mathrm{h}$ has analysis in the Figures 3 and 4). The relation between $q_{r}, q_{m}$, and $C_{o}$ is shown in Figure 7 after adjustment of $V_{o}$. As shown in Figure 7, the interference of on-ramp vehicle is increasing with the increasing of $V_{c}$ and the flow of on-ramp that has great influence is corresponding decreasing with the increasing of $V_{c}$. This is consistent with the actual situation, because the interference of lane-changing behavior of onramp vehicle to the flow of mainline is increasing with the increasing of $V_{c}$.

\section{Conclusions}

Upon the completion of the thesis, the following conclusions were drawn. 


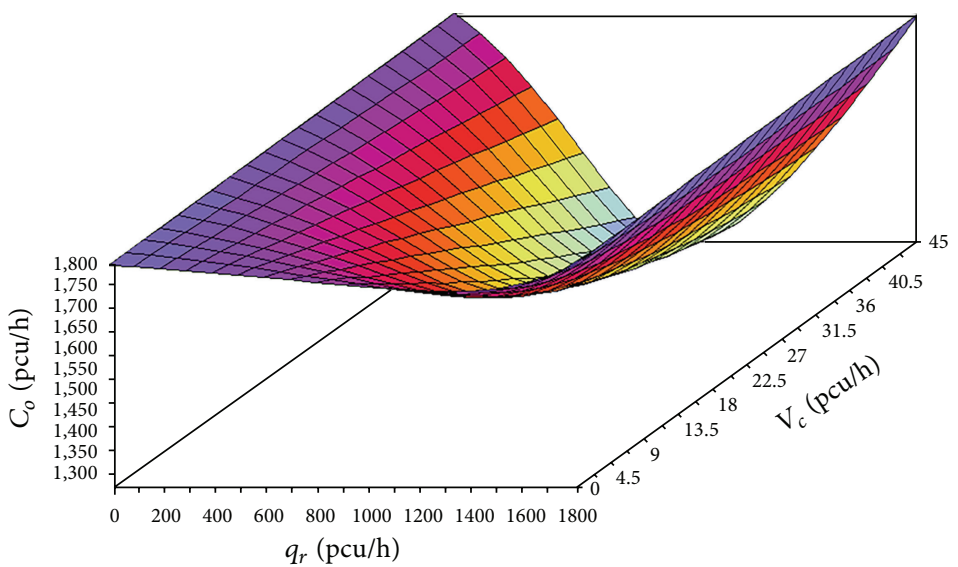

(a) $1800 \mathrm{pcu} / \mathrm{h}$

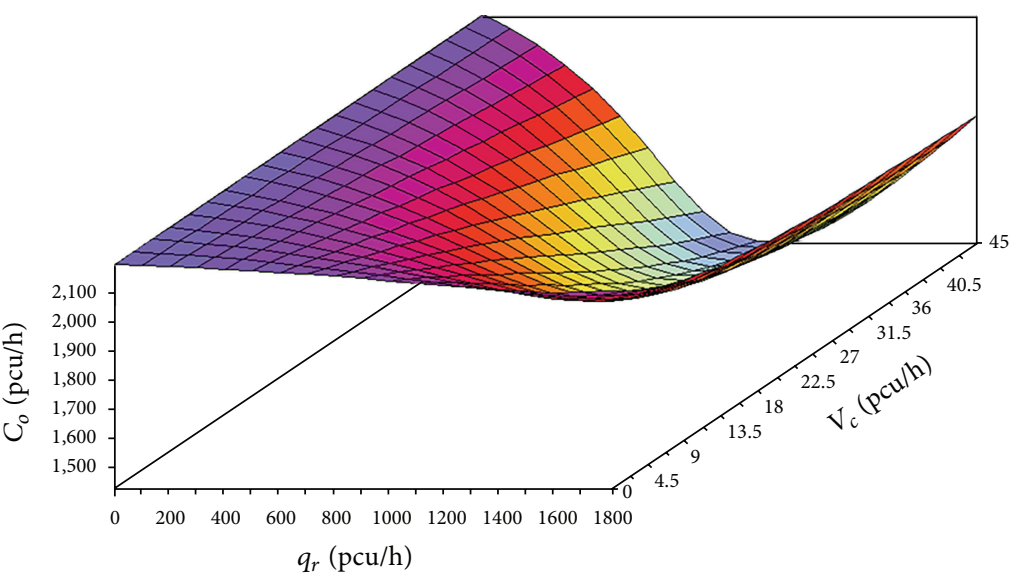

(b) $2200 \mathrm{pcu} / \mathrm{h}$

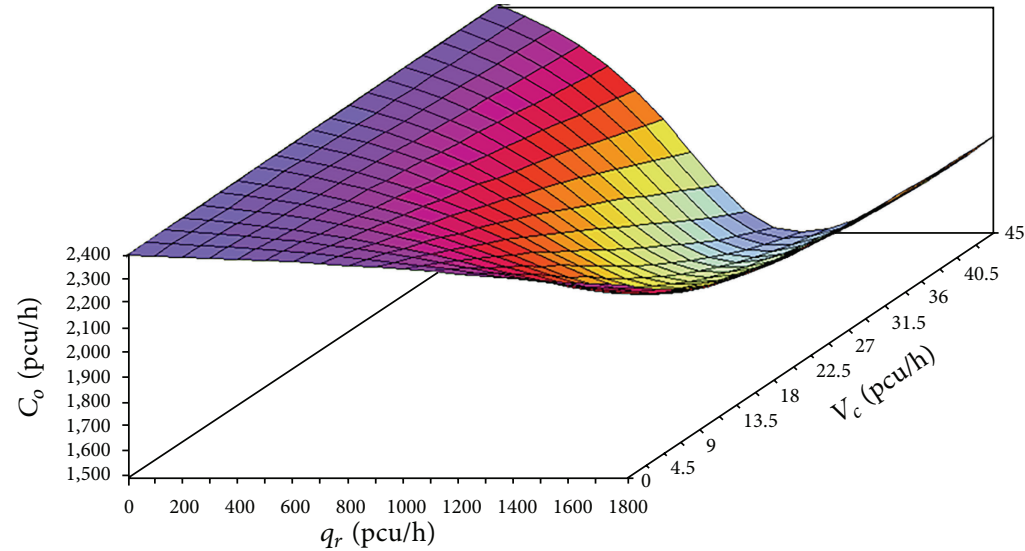

(c) $2400 \mathrm{pcu} / \mathrm{h}$

Figure 6: The relation diagram between $q_{r}, V_{c}$, and $C_{o}$ about different $C_{o}$ values.

(1) The capacity of on-ramp merging section is still a theoretical notion that lacks an unambiguous capacity value; this paper is to address this issue by studying its characteristics under different flow conditions using one week 5 min loop-detector data at Shanghai expressway system.
(2) This study puts forward three parameters: optimal time headway, time headway loss, and interference quantity of lane-changing. The capacity models for on-ramp merging section based on time headway loss were developed as a function of volume ratios from both of the mainline and the ramp, the gap between 


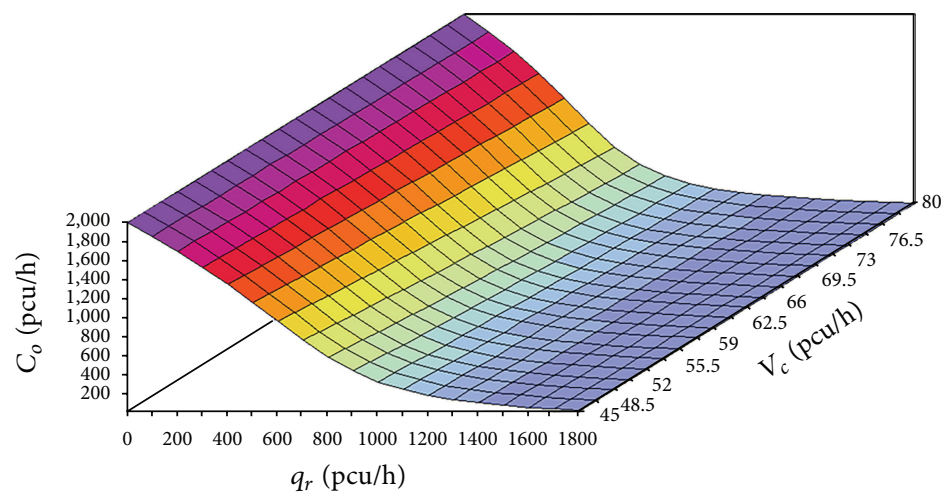

(a) The relation diagram between $q_{r}$ and $q_{m}$

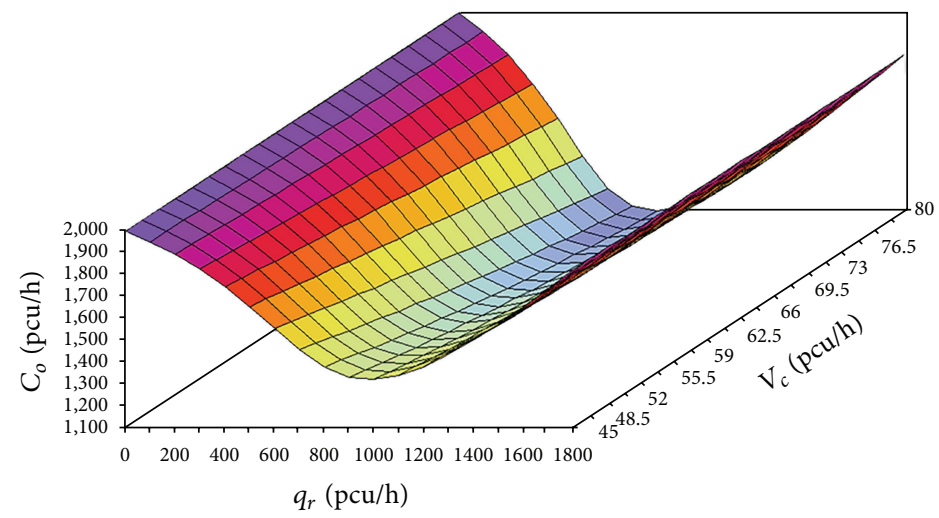

(b) The relation diagram between $q_{r}$ and $C_{o}$

Figure 7: The relation between $q_{r}, q_{m}$, and $C_{o}$ after $V_{c}$ adjustment.

optimal speed and the lane-changing speed of fleet. The capacity value changes depending on traffic flow situation and geometric factors.

(3) The drop of merge capacity is caused by driver's lanechanging maneuvers that frequently lead to vehicle variable speed and thereby time headway was extended relative to the minimum time headway that corresponds to the maximum capacity; the reduction of capacity is calculated by accumulative loss of time headway.

(4) Results compared with real data and microsimulation of on-ramp merging section show that this model produces reliable and accurate results, and the results meet the sensitivity analysis.

(5) Our studies results suggest that a big gap of speed between mainline and ramp vehicle should be reduced and the introduction of an accelerating lane long enough can remit this problem and improve the capacity of the on-ramp merging section.

(6) To improve the ramp control strategies can create smaller capacity drops, when compared with no control case that is unable to prevent excessive vehicle into the mainline by the ramp.
(7) Introducing the design of lane number balance (the number of mainline lanes on the downstream of ramp is equal to the sum of ramp lanes and mainline lanes on the upstream) can increasing the capacity of onramp merging section by decreasing driver's lanechanging maneuvers.

(8) This study about on-ramp merging section capacity of urban expressway is important for transportation planning, design, and operations.

\section{Conflict of Interests}

The authors declare that there is no conflict of interests regarding the publication of this paper.

\section{Acknowledgments}

This research is supported by the Central South University Science Fund Project, Hunan province, China, the Science and Technology Plans Project of Hunan province, Hunan province, China, and the Open Fund of Hunan Province University Key Laboratory of Bridge Engineering (Changsha University of Science and Technology), Hunan province, China. 


\section{References}

[1] M. J. Cassidy and J. Rudjanakanoknad, "Increasing the capacity of an isolated merge by metering its on-ramp," Transportation Research B, vol. 39, no. 10, pp. 896-913, 2005.

[2] J. A. Laval and C. F. Daganzo, "Lane-changing in traffic streams," Transportation Research B, vol. 40, no. 3, pp. 251-264, 2006.

[3] K. Chung, J. Rudjanakanoknad, and M. J. Cassidy, "Relation between traffic density and capacity drop at three freeway bottlenecks," Transportation Research B, vol. 41, no. 1, pp. 82-95, 2007.

[4] S. Oh and H. Yeo, "Microscopic analysis on the causal factors of capacity drop in highway merging sections," in Proceedings of the Transportation Research Board 91st Annual Meeting, Washington, DC, USA, 2012.

[5] A. Duret, J. Bouffier, and C. Buisson, "Onset of congestion from low-speed merging maneuvers within free-flow traffic stream," Transportation Research Record, no. 2188, pp. 96-107, 2010.

[6] B. Coifman and S. Kim, "Extended bottlenecks, the fundamental relationship, and capacity drop on freeways," Transportation Research A, vol. 45, no. 9, pp. 980-991, 2011.

[7] D. R. Drew, "Gap acceptance in the freeway merging process," Highway Research Record 208, 1967.

[8] S. Westphal, "Capacity of freeway on-ramps on German motorways," in Proceeding of the 2nd International Symposium on Highway Capacity, pp. 707-715, Sydney, Australia, 1994.

[9] P. Lertworawanich and L. Elefteriadou, "A methodology for estimating capacity at ramp weaves based on gap acceptance and linear optimization," Transportation Research B, vol. 37, no. 5, pp. 459-483, 2003.

[10] S. G. Kim and Y. Son, "Development of new merge capacity model and effects of ramp flow on the merge capacity," in Proceedings of the 82nd Annual Meeting of the Transportation Research Board, pp. 1-19, Washington, DC, USA, 2003.

[11] L. Leclercq, J. A. Laval, and N. Chiabaut, "Capacity drops at merges: an endogenous model," Transportation Research B, vol. 45, no. 9, pp. 1302-1313, 2011.

[12] M. R. Lorenz and L. Elefteriadou, "Defining freeway capacity as function of breakdown probability," Transportation Research Record, no. 1776, pp. 43-51, 2007.

[13] W. Brilon, J. Geistefeldt, and H. Zurlinden, "Implementing the concept of reliability for highway capacity analysis," Transportation Research Record, no. 2027, pp. 1-8, 2007.

[14] C. Zhao, W. Wang, and W. Li, "On-ramp capacity under different flow rate of major stream," Journal of Highway and Transportation Research and Development, vol. 22, no. 2, pp. 8285, 2005.

[15] Y. Ci, L. Wu, Y. Pei, and X. Ling, "Gap acceptance capacity model for on-ramp junction of urban freeway," Journal of Transportation Systems Engineering and Information Technology, vol. 9, no. 4, pp. 116-119, 2009. 


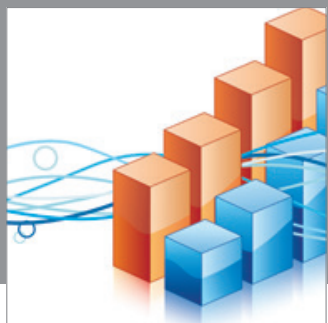

Advances in

Operations Research

mansans

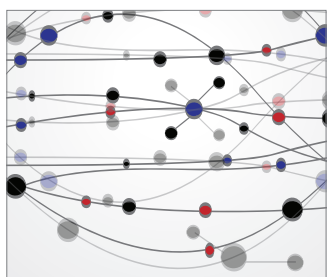

The Scientific World Journal
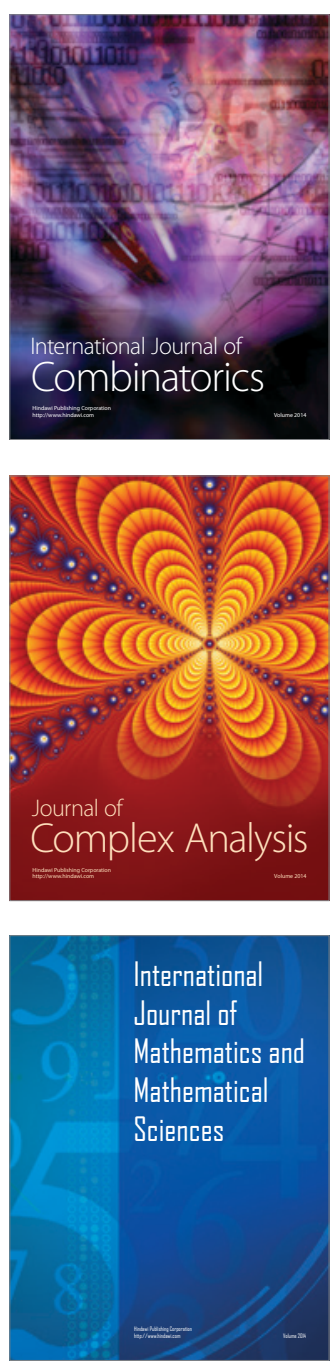
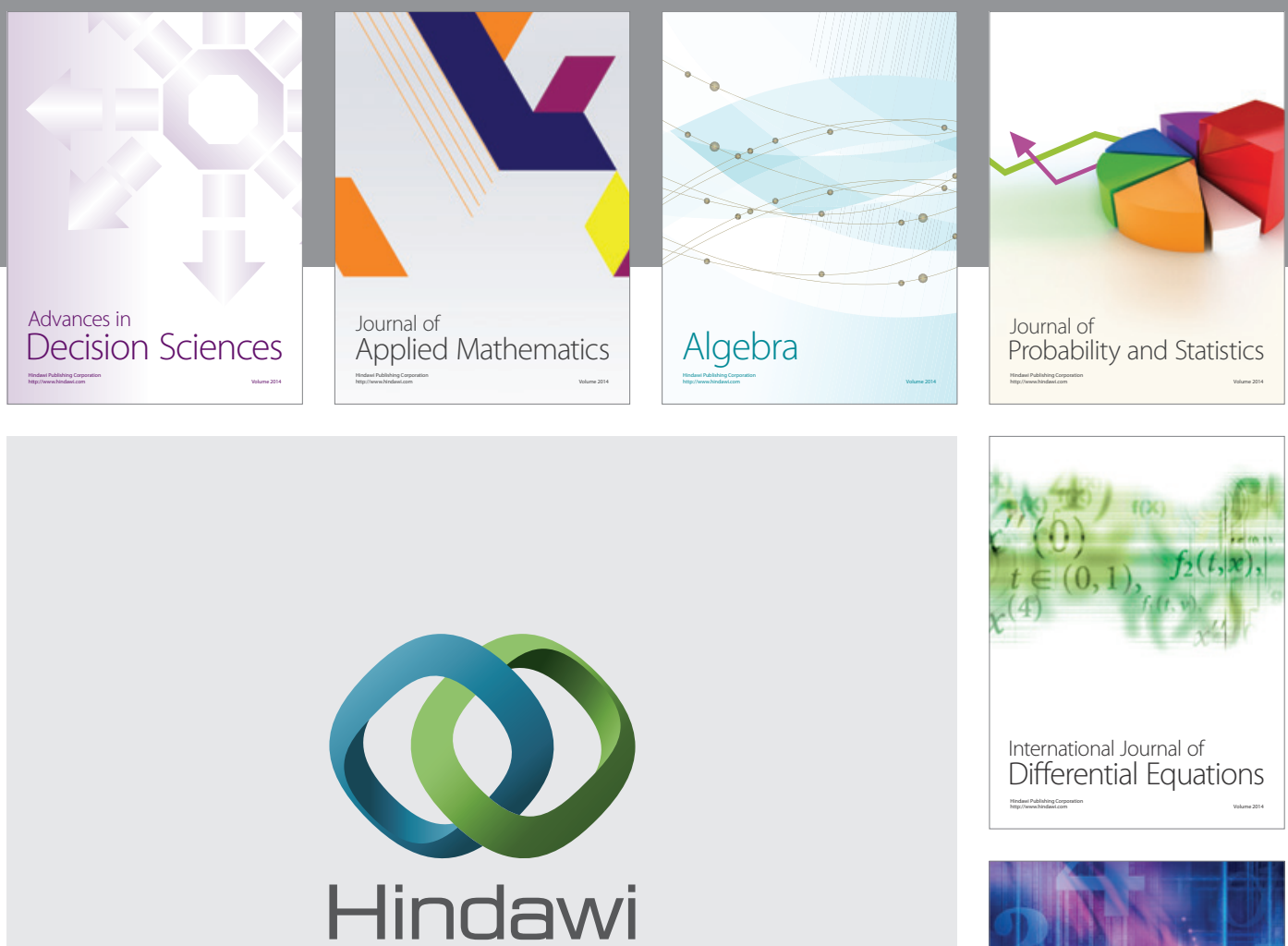

Submit your manuscripts at http://www.hindawi.com
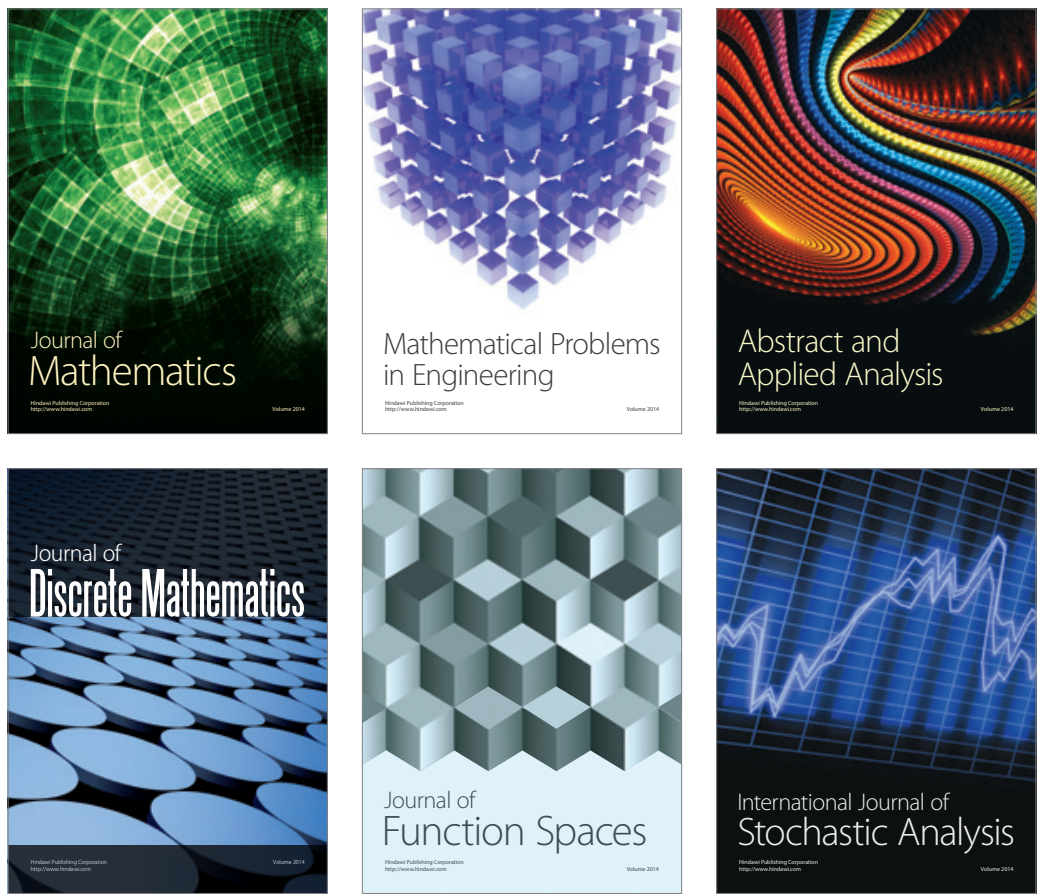

Journal of

Function Spaces

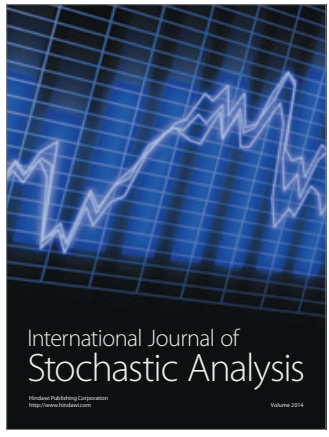

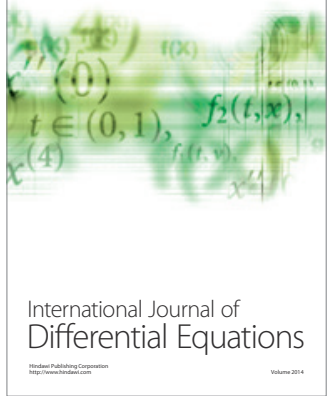
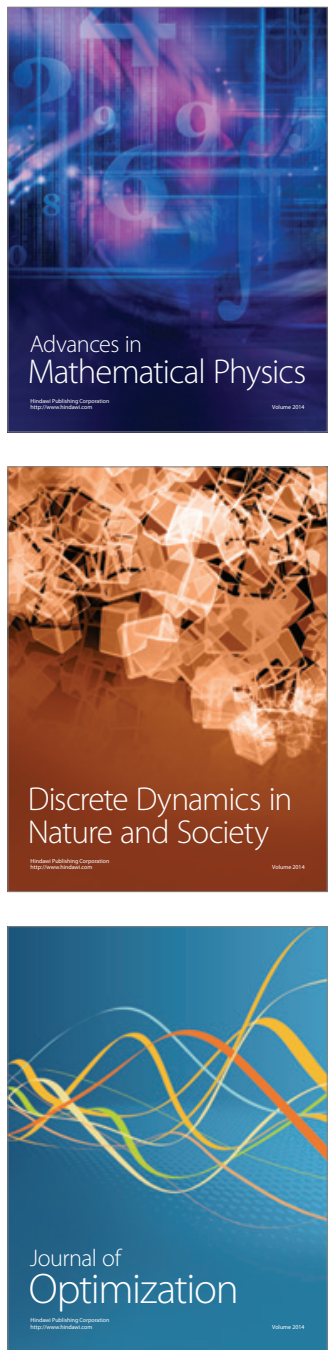